\title{
Integral Averages of Two Generalizations of the Poisson Kernel by Haruki and Rassias
}

\author{
Serap Bulut \\ Kocaeli University, Civil Aviation College, Arslanbey Campus, 41285 Izmit, Turkey \\ Correspondence should be addressed to Serap Bulut, bulutserap@yahoo.com \\ Received 15 November 2007; Revised 13 January 2008; Accepted 17 January 2008 \\ Recommended by John Rassias
}

In 1997, Haruki and Rassias introduced two generalizations of the Poisson kernel in two dimensions and discussed integral formulas for them. Furthermore, they presented an open problem. In 1999, Kim gave a solution to that problem. Here, we give a solution to this open problem by means of a different method. The purpose of this paper is to give integral averages of two generalizations of the Poisson kernel, that is, we generalize the open problem.

Copyright (C) 2008 Serap Bulut. This is an open access article distributed under the Creative Commons Attribution License, which permits unrestricted use, distribution, and reproduction in any medium, provided the original work is properly cited.

\section{Introduction}

It is well known that the Poisson kernel in two dimensions is defined by

$$
P(r, \theta) \stackrel{\text { def }}{=} \frac{1-r^{2}}{\left(1-r e^{i \theta}\right)\left(1-r e^{-i \theta}\right)},
$$

and the integral formula

$$
\frac{1}{2 \pi} \int_{0}^{2 \pi} P(r, \theta) d \theta=1
$$

holds. Here $r$ is a real parameter satisfying $|r|<1$.

In [1], Haruki and Rassias introduced two generalizations of the Poisson kernel.

The first generalization is defined by

$$
Q(\theta ; a, b) \stackrel{\text { def }}{=} \frac{1-a b}{\left(1-a e^{i \theta}\right)\left(1-b e^{-i \theta}\right)},
$$

where $a, b$ are complex parameters satisfying $|a|<1$ and $|b|<1$. 
The second generalization is defined by

$$
R(\theta ; a, b, c, d)=\frac{L(a, b, c, d)}{\left(1-a e^{i \theta}\right)\left(1-b e^{-i \theta}\right)\left(1-c e^{i \theta}\right)\left(1-d e^{-i \theta}\right)}
$$

where $a, b, c, d$ are complex parameters satisfying $|a|<1,|b|<1,|c|<1$, and $|d|<1$ as well as

$$
L(a, b, c, d) \stackrel{\text { def }}{=} \frac{(1-a b)(1-a d)(1-b c)(1-c d)}{1-a b c d} .
$$

Then they proved the integral formulas

$$
\begin{gathered}
\frac{1}{2 \pi} \int_{0}^{2 \pi} Q(\theta ; a, b) d \theta=1 \\
\frac{1}{2 \pi} \int_{0}^{2 \pi} R(\theta ; a, b, c, d) d \theta=1
\end{gathered}
$$

Remark 1.1. If we set $c=a$ and $d=b$ in (1.7), then we obtain

$$
\frac{1}{2 \pi} \int_{0}^{2 \pi} Q(\theta ; a, b)^{2} d \theta=\frac{1+a b}{1-a b} .
$$

Afterwards, they set the following definition and open problem.

For $n=0,1,2, \ldots$, let

$$
I_{n} \stackrel{\text { def }}{=} \frac{1}{2 \pi} \int_{0}^{2 \pi} Q(\theta ; a, b)^{n+1} d \theta
$$

where $a, b$ are complex parameters satisfying $|a|<1$ and $|b|<1$.

Open Problem 1.2. Compute $I_{n}$ for $n=2,3,4, \ldots$

In [2], Kim gave a solution to this open problem using the Laurent series expansion.

In the next section, we give a solution to the open problem by means of the Leibniz rule.

\section{A different solution of the open problem}

Theorem 2.1. It holds that

$$
I_{n}=\sum_{k=0}^{n} \frac{(n+k) !}{(n-k) !(k !)^{2}}\left(\frac{a b}{1-a b}\right)^{k}
$$

where $I_{n}$ is defined by (1.9).

Proof. We have

$$
I_{n}=\frac{1}{2 \pi} \int_{0}^{2 \pi}\left(\frac{1-a b}{\left(1-a e^{i \theta}\right)\left(1-b e^{-i \theta}\right)}\right)^{n+1} d \theta=\frac{1}{2 \pi} \int_{0}^{2 \pi} \frac{\left((1-a b) /\left(1-a e^{i \theta}\right)\right)^{n+1}}{\left(1-b e^{-i \theta}\right)^{n+1}} d \theta .
$$


By the change of variables $z=e^{i \theta}$ and setting

$$
f(z) \stackrel{\text { def }}{=}\left(\frac{1-a b}{1-a z}\right)^{n+1} z^{n}
$$

we have

$$
I_{n}=\frac{1}{2 \pi i} \int_{|z|=1} \frac{f(z)}{(z-b)^{n+1}} d z,
$$

where the complex integral of the function $f(z)$ along the unit circle $|z|=1$ is in the positive direction.

Since $f(z)$ is an analytic function in $|z| \leq 1$, by Cauchy's integral formula for the derivative, we obtain

$$
I_{n}=\frac{f^{(n)}(b)}{n !} .
$$

So we must calculate $f^{(n)}(z)$. For this purpose, we will use the Leibniz rule (generalized product rule).

Let

$$
\begin{gathered}
g(z) \stackrel{\text { def }}{=} z^{n}, \\
h(z) \stackrel{\text { def }}{=}(1-a z)^{-(n+1) .} .
\end{gathered}
$$

Thus by (2.3) and (2.6), we have

$$
f(z)=(1-a b)^{n+1} g(z) h(z) .
$$

Applying the Leibniz rule to (2.7), we get

$$
\begin{aligned}
f^{(n)}(z) & =(1-a b)^{n+1}(g h)^{(n)}(z) \\
& =(1-a b)^{n+1} \sum_{k=0}^{n}\left(\begin{array}{l}
n \\
k
\end{array}\right) g^{(n-k)}(z) h^{(k)}(z) \\
& =n !(1-a b)^{n+1} \sum_{k=0}^{n} \frac{(n+k) !}{(n-k) !(k !)^{2}}(a z)^{k}(1-a z)^{-(n+k+1),}
\end{aligned}
$$

where

$$
\begin{gathered}
g^{(n-k)}(z)=\frac{n !}{k !} z^{k} \\
h^{(k)}(z)=a^{k} \frac{(n+k) !}{n !}(1-a z)^{-(n+k+1)} .
\end{gathered}
$$

If we take $z=b$ in (2.8), we obtain

$$
\frac{f^{(n)}(b)}{n !}=\sum_{k=0}^{n} \frac{(n+k) !}{(n-k) !(k !)^{2}}\left(\frac{a b}{1-a b}\right)^{k} .
$$

Thus by (2.5) and (2.10), we get the desired result. 


\section{New generalizations of the open problem}

In [3], the authors gave the values of the integral

$$
\frac{1}{2 \pi} \int_{0}^{2 \pi} P^{n+1}(r, \theta) d \theta
$$

for all real $n>-1$.

In this section, we will generalize $I_{n}$, and hence above integral as follows.

Theorem 3.1 (Main theorem). For any real number $u$, it holds that

$$
J_{u}:=\frac{1}{2 \pi} \int_{0}^{2 \pi} Q(\theta ; a, b)^{u} d \theta=(1-a b)^{u}{ }_{2} F_{1}(u, u ; 1 ; a b),
$$

where ${ }_{2} F_{1}$ is the usual hypergeometric function.

Proof. Let $u$ be any real number. Define the shifted factorial (or the Pochhammer symbol) by

$$
(u)_{k}:=\frac{\Gamma(u+k)}{\Gamma(u)} \quad(u \neq-n, n=0,1,2, \ldots),
$$

where $\Gamma$ is the gamma function. If $u=-n$ is a nonpositive integer, define $(-n)_{k}:=(-n)(-n+$ 1) $\cdots(-n+k-1)$ so that $(-n)_{k}=0$ for $k=n+1, n+2, \ldots$ Then

$$
\frac{1}{(1-w)^{u}}=\sum_{k=0}^{\infty} \frac{(u)_{k}}{k !} w^{k} \quad(|w|<1) .
$$

For $z=e^{i \theta}$, one computes that

$$
\begin{aligned}
J_{u} & =\frac{1}{2 \pi} \int_{0}^{2 \pi} Q(\theta ; a, b)^{u} d \theta=\frac{1}{2 \pi} \int_{0}^{2 \pi} \frac{(1-a b)^{u}}{\left(1-a e^{i \theta}\right)^{u}\left(1-b e^{-i \theta}\right)^{u}} d \theta \\
& =\frac{(1-a b)^{u}}{2 \pi i} \int_{|z|=1} \frac{d z}{z(1-a z)^{u}(1-b / z)^{u}} \\
& =\frac{(1-a b)^{u}}{2 \pi i} \int_{|z|=1} \frac{1}{z}\left(\sum_{k=0}^{\infty} \frac{(u)_{k}}{k !} a^{k} z^{k}\right)\left(\sum_{l=0}^{\infty} \frac{(u)_{l}}{l !} \frac{b^{l}}{z^{l}}\right) d z .
\end{aligned}
$$

The integral of the terms with $k \neq l$ is 0 by residue theorem, and thus

$$
J_{u}=(1-a b)^{u} \sum_{k=0}^{\infty} \frac{(u)_{k}(u)_{k}}{(1)_{k} k !}(a b)^{k}=(1-a b)^{u}{ }_{2} F_{1}(u, u ; 1 ; a b),
$$

where ${ }_{2} F_{1}$ is the usual hypergeometric function. 
It is routine to check that

$$
J_{1}=1, \quad J_{2}=\frac{1+a b}{1-a b}
$$

as obtained in [1] because, then, the series above is summable via elementary functions. Also for $n=0,1,2, \ldots$, one has

$$
\begin{aligned}
& J_{n}=(1-a b)^{n} \sum_{k=0}^{\infty}\left(\begin{array}{c}
n-1+k \\
k
\end{array}\right)^{2}(a b)^{k}, \\
& J_{-n}=\frac{1}{(1-a b)^{n}} \sum_{k=0}^{n}\left(\begin{array}{l}
n \\
k
\end{array}\right)^{2}(a b)^{k} .
\end{aligned}
$$

Moreover, setting $a=b=r$ generalizes the results of [3] to all real powers $u$ of the Poisson kernel.

The same method applied to the integral averages of the second generalization of the Poisson kernel yields

$$
K_{u}:=\frac{1}{2 \pi} \int_{0}^{2 \pi} R(\theta ; a, b, c, d)^{u} d \theta=L(a, b, c, d)^{u} \sum_{j+l=k+m} \frac{(u)_{j}(u)_{k}(u)_{l}(u)_{m}}{j ! k ! ! ! m !} a^{j} b^{k} c^{l} d^{m} .
$$

There is a further connection with the fractional-order derivative in [3] which is called $D^{u}$ here for any real number $u$. If $p$ is also any real number, let $m=\lceil p\rceil$ be the least integer greater than or equal to $p$. Then one can compute with $s=t / x$ that

$$
\begin{aligned}
D^{u}\left(x^{p}\right) & =\frac{d^{m}}{d x^{m}}\left[\frac{1}{\Gamma(m-u)} \int_{0}^{x}(x-t)^{m-u-1} t^{p} d t\right] \\
& =\frac{d^{m}}{d x^{m}}\left[\frac{x^{m-u+p}}{\Gamma(m-u)} \int_{0}^{1}(1-s)^{m-u-1} s^{p} d s\right] \\
& =\frac{d^{m}}{d x^{m}}\left[\frac{x^{m-u+p}}{\Gamma(m-u)} \mathrm{B}(m-u, p+1)\right] \\
& =\frac{d^{m}}{d x^{m}}\left[\frac{\Gamma(p+1)}{\Gamma(m-u+p+1)} x^{m-u+p}\right]=\frac{x^{p-u}}{(p+1)_{-u}},
\end{aligned}
$$

which agrees with the usual derivative when $u$ is a positive integer, where B is the beta function, $u \neq p+1, p+2, \ldots$, and $p \neq 0,-1,-2, \ldots$.

If $u \neq 0,-1,-2, \ldots$, then

$$
\frac{1}{\Gamma(u)^{2}} D^{u-1}\left(x^{u-1} D^{u-1}\left(\frac{x^{u-1}}{1-x}\right)\right)=\frac{1}{\Gamma(u)^{2}} D^{u-1}\left(x^{u-1} D^{u-1}\left(\sum_{k=0}^{\infty} x^{k+u-1}\right)\right)=\sum_{k=0}^{\infty} \frac{(u)_{k}^{2}}{(k !)^{2}} x^{k}
$$

by successively applying the above fractional differentiation formula. Thus

$$
J_{u}=\left.\frac{(1-x)^{u}}{\Gamma(u)^{2}} D^{u-1}\left(x^{u-1} D^{u-1}\left(\frac{x^{u-1}}{1-x}\right)\right)\right|_{x=a b} .
$$




\section{Acknowledgment}

The author is grateful to the referee for useful comments and suggestions.

\section{References}

[1] H. Haruki and T. M. Rassias, "New generalizations of the Poisson kernel," Journal of Applied Mathematics and Stochastic Analysis, vol. 10, no. 2, pp. 191-196, 1997.

[2] B. Kim, "The solution of an open problem given by H. Haruki and T. M. Rassias," Journal of Applied Mathematics and Stochastic Analysis, vol. 12, no. 3, pp. 261-263, 1999.

[3] A. S. Galbraith and J. W. Green, "A note on the mean value of the Poisson kernel," Bulletin of the American Mathematical Society, vol. 53, pp. 314-320, 1947. 


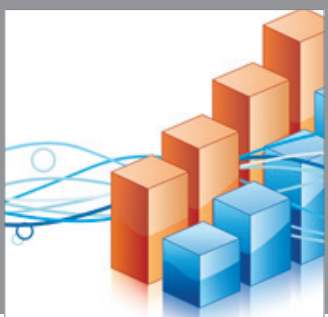

Advances in

Operations Research

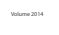

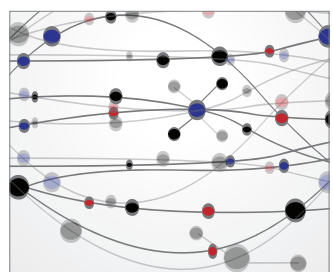

\section{The Scientific} World Journal
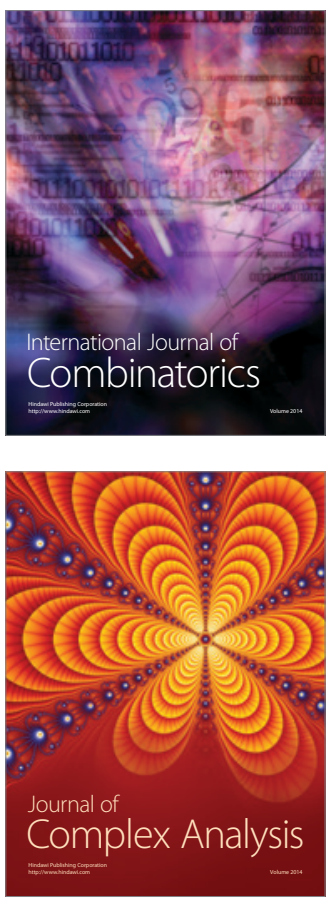

International Journal of

Mathematics and

Mathematical

Sciences
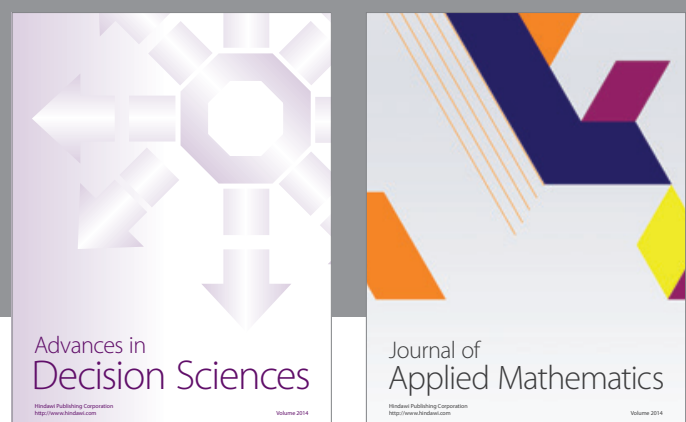

Journal of

Applied Mathematics
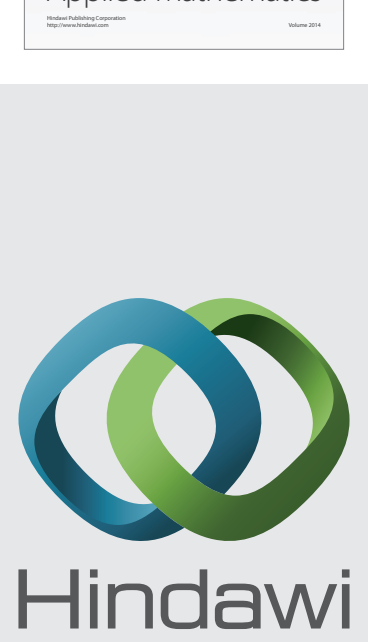

Submit your manuscripts at http://www.hindawi.com
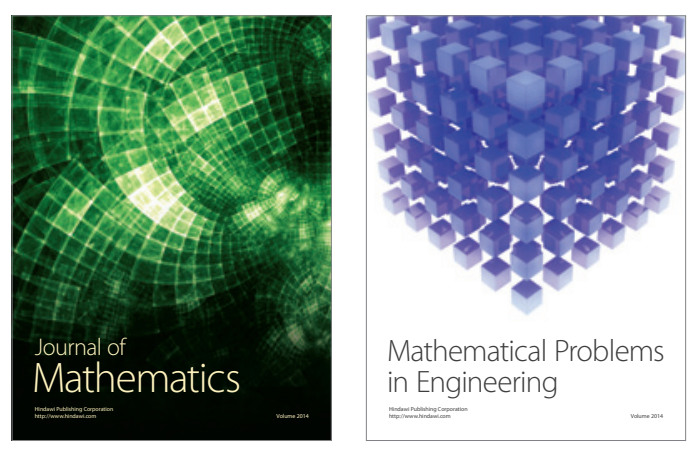

Mathematical Problems in Engineering
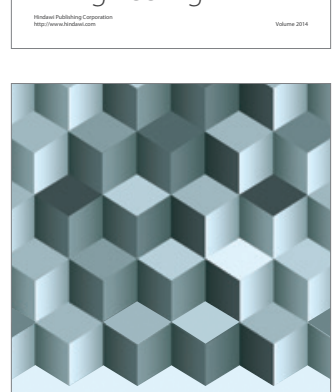

Journal of

Function Spaces
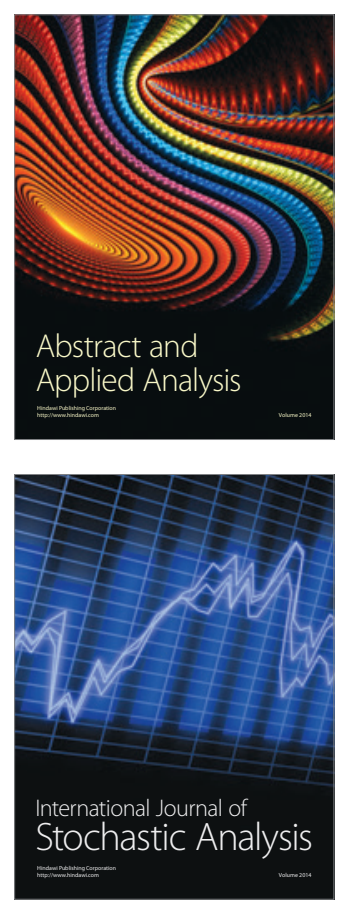

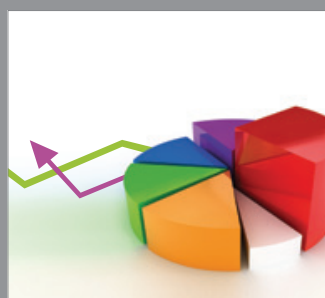

ournal of

Probability and Statistics

Promensencen
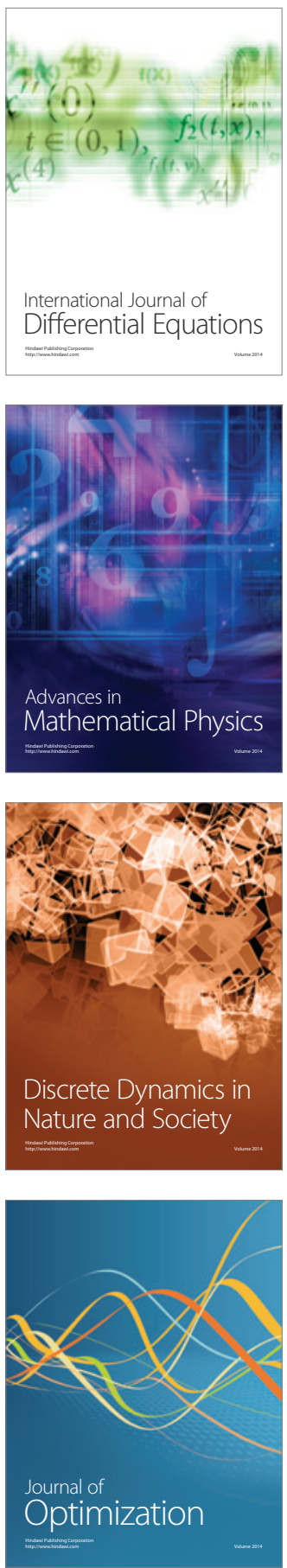